\title{
The equational complexity of Lyndon's algebra
}

\author{
Marcel Jackson and George F. McNulty \\ In memory of George's friend Buddy
}

\begin{abstract}
The equational complexity of Lyndon's nonfinitely based 7-element algebra lies between $n-4$ and $2 n+1$. This result is based on a new algebraic proof that Lyndon's algebra is not finitely based. We prove that Lyndon's algebra is inherently nonfinitely based relative to a rather rich class of algebras. We also show that the variety generated by Lyndon's algebra contains subdirectly irreducible algebras of all cardinalities except 0,1 , and 4 .
\end{abstract}

\section{Introduction}

In 1954 Roger Lyndon [18] published the earliest example of a finite algebra $\mathbf{L}$ that is not finitely based. Zoltán Székely noticed that Lyndon's algebra arises from a finite automaton and can be conveniently displayed by a diagram. Figure 1 gives the diagrams of two such algebras. The elements of the algebra $\mathbf{L}$

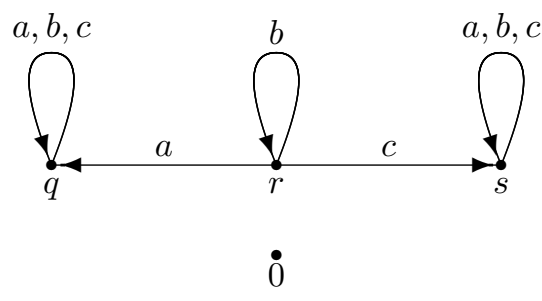

$\mathbf{L}$

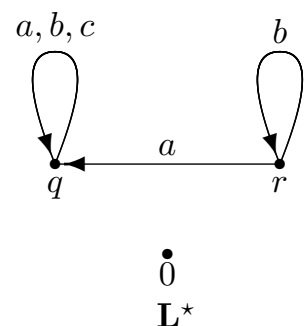

$\mathbf{L}^{\star}$

Figure 1. Two Automatic Algebras

fall into three pairwise disjoint sets: a set $\{q, r, s\}$ of states, a set $\{a, b, c\}$ of letters, and $\{0\}$. The sole basic operation of the algebra, which is binary and written here as juxtaposition, comes from the transition function of the underlying automaton and can be read from the diagram. For example we

Presented by E. Kiss.

Received January 7, 2010; accepted in final form September 8, 2010.

2010 Mathematics Subject Classification: Primary: 08B05 Secondary: 03C05, 68Q17.

Key words and phrases: Equational complexity; variety of algebras; relatively inherently nonfinitely based; shift automorphism method; Lyndon's algebra; automatic algebra; finite algebra membership problem.

The first author was partially supported by ARC Discovery Project Grants DP0342459 and DP1094578. 
have $q=a r$ and $r=b r$. Products, like $a c$ and $r b$, not associated in this way with the arrows in the diagram take the default value 0 . More precisely, a groupoid $\mathbf{A}$ is automatic provided $A$ is the union of three pairwise disjoint sets $\Sigma, Q$, and $\{0\}$ and the operation, written as juxtaposition, conforms to the constraint

- $a b=0$ or

- $a \in \Sigma, b \in Q$, and $a b \in Q$, for all $a, b \in A$.

The elements of $Q$ are referred to as states, while the elements of $\Sigma$ are referred to as letters.

The algebra $\mathbf{L}$ is essentially the algebra put forward in Lyndon's paper. (Actually, Lyndon's original algebra took 0 - which can be defined by the term $x x$ - as a distinguished constant and wrote the operation in the opposite order.) In 2008, Edmond W. H. Lee [17] proved that the subdirectly irreducible algebra $\mathbf{L}^{\star}$ generates the same variety as $\mathbf{L}$.

Lyndon's algebra has a number of interesting properties. Tamás Bajusz, George McNulty and Ágnes Szendrei [1] showed that it fails to be inherently nonfinitely based. Keith Kearnes and Ross Willard [14] observed that each automatic algebra, including $\mathbf{L}$ and $\mathbf{L}^{\star}$, is 2-step strongly solvable. Lee [17] proved that the variety generated by $\mathbf{L}$ is of finite height and each of its proper subvarieties is a Cross variety (and hence finitely based).

The main point of the present paper is to offer tight estimates on the equational complexity of Lyndon's algebra.

The equational complexity of a variety $\mathcal{V}$ of finite signature is the function $\beta_{\mathcal{V}}$ from the positive integers into the natural numbers so that $\beta_{\mathcal{V}}(n)$ is the smallest natural number $\ell$ such that any algebra $\mathbf{B}$ of cardinality less than $n$ belongs to $\mathcal{V}$ if and only if each equation of length less than $\ell$ that is true in $\mathcal{V}$ is also true in $\mathbf{B}$. Thus the equational complexity function measures how much of the equational theory of $\mathcal{V}$ must be tested to see if $\mathbf{B}$ belongs to $\mathcal{V}$. If $\mathcal{V}$ is the variety generated by an algebra $\mathbf{A}$, we use $\beta_{\mathbf{A}}$ in place of $\beta_{\mathcal{V}}$. The equational complexity function was first introduced and investigated in Székely's dissertation [28]. Further developments were made by Gábor Kun and Vera Vertési [16], by Marcin Kozik [15], and by McNulty, Willard, and Székely [20].

In 1988 Mark Sapir [27] observed, in essence, that the equational complexity of any finite algebra that is not finitely based but that fails to be inherently nonfinitely based (as holds for Lyndon's algebra) must eventually dominate every constant function. (See also the paper of Robert Cacioppo [5].) McNulty, Willard and Székely [20] showed that $\beta_{\mathbf{L}}(n)$ is dominated by $12 n-20$ and left as an open problem whether $\beta_{\mathbf{L}}$ eventually dominates some strictly increasing linear function. That problem is solved here. We show that

$$
n-4<\beta_{\mathbf{L}}(n) \leq 2 n+1
$$

for all $n>3$. 
Let $\mathcal{P}$ be a property of varieties. We say that a variety $\mathcal{V}$ is inherently nonfinitely based relative to $\mathcal{P}$ provided that, if $\mathcal{V}$ and $\mathcal{U}$ are varieties with property $\mathcal{P}$ and $\mathcal{V} \subseteq \mathcal{U}$, then $\mathcal{U}$ is not finitely based. When $\mathcal{P}$ is the property of being locally finite, we obtain the notion of inherently nonfinitely based variety. When $\mathcal{P}$ is the property of being finitely generated, we obtain the notion of strongly nonfinitely based variety (see Volkov [30]). This concept, framed here for varieties, made its first appearance in the context of quasivarieties in the recent paper of Jackson and Volkov [12].

Let $\mathcal{W}$ be a variety. We say that the variety $\mathcal{V}$ is inherently nonfinitely based relative to $\mathcal{W}$ provided that, if $\mathcal{U}$ is a variety such that $\mathcal{V} \subseteq \mathcal{U} \subseteq \mathcal{W}$, then $\mathcal{U}$ is not finitely based. This is the special case of the notion described in the preceding paragraph when $\mathcal{P}$ is taken to be "is included in $\mathcal{W}$ ". Roughly, the richer the class $\mathcal{W}$ is, the stronger the nonfinite basis property for $\mathcal{V}$ is.

The present paper provides another proof that $\mathbf{L}$ is not finitely based. Lyndon's proof, which occupies less than two pages, is syntactical in nature. The proof offered here has an algebraic character. Indeed, we can show that Lyndon's algebra is actually inherently nonfinitely based relative to the variety generated by all automatic algebras - an observation Lyndon might also have drawn from his proof.

We also prove that the variety generated by $\mathbf{L}$ contains subdirectly irreducible algebras of all cardinalities, except 0,1 , and 4 , and it has none of these cardinalities. In 1976, Park [23] proved that there are arbitrarily large finite, as well as arbitrarily large infinite, subdirectly irreducible algebras in this variety. Finally, we find that Lyndon's algebra provides an example that a key hypothesis of the Shift Automorphism Theorem [2] cannot be eliminated.

1.1. Prospects for Further Development. While the present article is centered on Lyndon's algebra, we frame here some broader directions for further research that suggested themselves to us during the course of our work.

Let $\mathbf{A}$ be a finite algebra of finite signature. Consider the implications:

$\mathbf{A}$ is inherently nonfinitely based in the finite sense

That is, if $\mathbf{A} \in \mathcal{U}$ and $\mathcal{U}$ is a finitely based variety, then there is a natural number $p$ so that $\mathcal{U}$ contains arbitrarily large finite $p$-generated algebras.

A is inherently nonfinitely based

That is, if $\mathbf{A} \in \mathcal{U}$ and $\mathcal{U}$ is a finitely based variety, then $\mathcal{U}$ is not locally finite.

$\Downarrow$

A is strongly nonfinitely based

That is, if $\mathbf{A} \in \mathcal{U}$ and $\mathcal{U}$ is a finitely based variety, then $\mathcal{U}$ is not generated by a finite algebra.

$\Downarrow$

A is not finitely based

That is, if $\mathbf{A} \in \mathcal{U}$ and $\mathcal{U}$ is a finitely based variety, then $\mathcal{U}$ is not generated

by $\mathbf{A}$. 
The proof given by Bajusz, McNulty, and Szendrei [1] actually constructs a finitely based finite algebra which has Lyndon's algebra $\mathbf{L}$ as a subalgebra. This demonstrates that the bottom arrow above cannot be reversed. We know of no similar examples to show that any of the other arrows above cannot be reversed. Volkov [30] in his Problem 4.4 raised the question of reversing the middle arrow, while the problem of reversing of the top arrow was raised in [20]. Discovering which, if any, of these arrows can be reversed merits investigation. For example, reversing the top arrow would settle a problem of Eilenberg and Schützenberger open since 1976, [8, 20]. A similar chain of implications can be made by replacing $\mathbf{A}$ by a locally finite variety $\mathcal{V}$ of finite signature. Reversing the arrows in this context is an interesting open problem, as well.

It is also possible to relativize the chain of implications above to a variety $\mathcal{W}$. For example, we call a finite algebra $\mathbf{A}$ strongly nonfinitely based relative to $\mathcal{W}$ provided $\mathcal{U}$ is not finitely based whenever $\mathcal{U}$ is a finitely generated variety such that $\mathbf{A} \in \mathcal{U} \subseteq \mathcal{W}$. Jackson [11] and Kadourek [13] contain examples of finite algebras $\mathbf{A}$ and finitely based varieties $\mathcal{W}$ so that $\mathbf{A}$ is strongly nonfinitely based relative to $\mathcal{W}$ but $\mathbf{A}$ fails to be inherently nonfinitely based. To set this in the context at the top of the previous page, we have taken $\mathcal{P}$ to be "the variety is included in $\mathcal{W}$ and is generated by a finite algebra." Similar adjustments might be made to each of the properties on our chain of implications.

We show in the present article that the equational complexity of Lyndon's algebra exhibits linear growth. We know of no finite algebra, apart from those which are finitely based, whose growth is bounded above by a properly sublinear function-for instance, by $\log \log (n)$. Should such finite algebras exist, they may be candidates to show that the various arrows above cannot be reversed.

The equational complexity of a variety $\mathcal{V}$ of finite signature is related to the computational complexity of the following problem.

The Finite Algebra Membership Problem for $\mathcal{V}$ Input: A finite algebra $\mathbf{B}$ of the same signature as $\mathcal{V}$.

Problem: Determine whether $\mathbf{B} \in \mathcal{V}$.

According to results in the final section of [20] the Finite Algebra Membership Problem for $\mathcal{V}$ is in the complexity class EXPTIME, provided $\beta_{\mathcal{V}}$ is bounded above by a polynomial. We observe that the proof there actually shows that it belongs to the complexity class PSPACE. Hence, the finite algebra membership problem for Lyndon's algebra $\mathbf{L}$ belongs to PSPACE.

Another line of reasoning gives what appears to be a somewhat better result. Suppose that $\mathcal{V}$ is generated by a finite algebra $\mathbf{A}$ of finite signature. It is easy to see that the equational theory of $\mathbf{A}$ is in coNP: the certificate required to reject an equation $s \approx t$ is just an assignment of elements of $A$ to the variables in $s \approx t$. Such an assignment is bounded by roughly the complexity of the equation. Let $\mathcal{O}$ be an oracle for the equational theory of $\mathbf{A}$. To reject a finite algebra $\mathbf{B}$ from membership in $\mathcal{V}$ we can use as a certificate an equation $s \approx t$, 
with length bounded by $\beta \mathcal{V}(|B|)$, and an assignment of elements of $B$ to the variables in $s \approx t$. We can check that the assignment invalidates the equation in polynomial time and we can determine that $s \approx t$ holds in $\mathcal{V}$ from the oracle $\mathcal{O}$. In this way we find that the finite algebra membership problem of $\mathcal{V}$ is in coNPO ; that is it can be settled in coNP time with the help of the coNP oracle $\mathcal{O}$. This means that the finite algebra membership problem for $\mathcal{V}$ lies in the complexity class $\Pi_{2}^{P}$, at the second level of the polynomial hierarchy. It is known that $\Pi_{2}^{P} \subseteq$ PSPACE and it may well be much smaller that PSPACE.

Below we show that the finite algebra membership problem of Lyndon's algebra actually belongs to $\mathrm{P}$; that is it is solvable in polynomial time.

In the present article we show that Lyndon's algebra is inherently nonfinitely based relative to the variety generated by all the automatic algebras. Other automatic algebras are known to be inherently nonfinitely based in the finite sense, for instance the one constructed by Ralph McKenzie in 1996 [19]. See [20] for further information. Still others are known to be finitely based, see Murski1's 1996 paper [21] and the paper of Lee [17]. It seems within reach to classify all the finitely based finite automatic algebras. One potential benefit of such a classification, coupled with a similar investigation of dualizability of automatic algebras, is that it could lead to a better understanding of the connection between dualizability and the finite basis property. For more on duality theory see the books of David Clark and Brian Davey [6] and of Jane Pitkethly and Brian Davey [24].

\section{Another proof that Lyndon's algebra is not finitely based}

Let $n$ be a natural number and $\mathcal{V}$ be a variety. We use $\mathcal{V}^{(n)}$ to denote the variety based on all the equations true in $\mathcal{V}$ such that all variables occurring in these equations are drawn from the set $\left\{x_{0}, \ldots, x_{n-1}\right\}$. Observe that an algebra $\mathbf{B} \in \mathcal{V}^{(n)}$ if and only if every subalgebra of $\mathbf{B}$ with $n$ or fewer generators belongs to $\mathcal{V}$. Birkhoff [3] observed that if $\mathcal{V}$ is a locally finite variety of finite signature, then $\mathcal{V}^{(n)}$ is finitely based, for every natural number $n$. It follows that a locally finite variety of finite signature fails to be finitely based if and only if $\mathcal{V}^{(n)} \neq \mathcal{V}$, for all natural numbers $n$. In more detail, we see that a locally finite variety of finite signature fails to be finitely based if and only if for each natural number $n$ there is an algebra $\mathbf{C}_{n}$ and an equation $\epsilon_{n}$ such that

(a) $\mathbf{C}_{n} \in \mathcal{V}^{(n)}$

(b) $\mathcal{V} \models \epsilon_{n}$, and

(c) $\epsilon_{n}$ fails in $\mathbf{C}_{n}$.

Observation. Let $\mathcal{V}$ be a locally finite variety of finite signature and suppose that $\mathcal{V}$ is not finitely based. Let $\mathcal{W}=\operatorname{Mod}\left\{\epsilon_{n} \mid n\right.$ is a natural number $\}$, where the equations $\epsilon_{n}$ are selected as described above. Then $\mathcal{V}$ is inherently nonfinitely based relative to $\mathcal{W}$. 
To see this, consider a variety $\mathcal{U}$ with $\mathcal{V} \subseteq \mathcal{U}$. Suppose that $\mathcal{U}=\operatorname{Mod} \Sigma$ where $\Sigma$ is a set of equations in which only finitely many distinct variables occur. It follows that $\mathcal{V}^{(m)} \subseteq \mathcal{U}$ for any sufficiently large $m$. As a consequence $\mathbf{C}_{m} \in \mathcal{U}$ for all large enough $m$. So it must be that $\mathcal{U} \nsubseteq \operatorname{Mod}\left\{\epsilon_{n} \mid\right.$ $n$ is a natural number $\}$.

Lyndon's Nonfinite Basis Theorem. The seven element automatic algebra $\mathbf{L}$ is not finitely based.

Proof. Let $\mathcal{V}$ be the variety generated by $\mathbf{L}$. To establish this theorem we will construct an algebra $\mathbf{C}_{n} \in \mathcal{V}^{(n)}$ so that $\mathbf{C}_{n} \notin \mathcal{V}$.

We render the elements of $\left(\mathbf{L}^{\star}\right)^{n}$, which are $n$-tuples, as words of length $n$. That is we use $a a b r c 0$ in place of $(a, a, b, r, c, 0)$. Let $\mathbf{A}_{n}$ be the subalgebra of $\left(\mathbf{L}^{\star}\right)^{n}$ generated by the following $n+1$ words:

$$
\begin{aligned}
& \beta_{n} \quad:=r \quad r \quad \ldots \quad r \quad r \quad r
\end{aligned}
$$

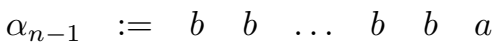

$$
\begin{aligned}
& \alpha_{n-2}:=\begin{array}{llllll}
b & b & \ldots & b & a & c
\end{array} \\
& \alpha_{n-2}:=b \quad b \quad \ldots \quad a \quad c \quad c \\
& \text { : } \\
& \alpha_{2} \quad:=\begin{array}{llllll}
b & b & \ldots & c & c & c
\end{array} \\
& \alpha_{1} \quad:=\begin{array}{llllll}
b & a & \ldots & c & c & c
\end{array} \\
& \alpha_{0} \quad:=\begin{array}{lllllllllllll} 
& a & c & \ldots & c & c & c
\end{array}
\end{aligned}
$$

For $i<n$ put $\beta_{i}:=\alpha_{i} \beta_{i+1}$. Hence

$$
\begin{aligned}
& \beta_{n-1}:=\alpha_{n-1} \beta_{n}=\begin{array}{lllllll}
r & r & \ldots & r & r & q
\end{array} \\
& \beta_{0} \quad:=\alpha_{0} \beta_{1}=\begin{array}{lllllll} 
& q & q & \ldots & q & q & q
\end{array}
\end{aligned}
$$

We need an additional element of $\left(\mathbf{L}^{\star}\right)^{n}$, namely

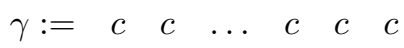

We let $\mathbf{A}_{n}^{\prime}$ be the subalgebra of $\left(\mathbf{L}^{\star}\right)^{n}$ generated by $\beta_{n}, \alpha_{n-1}, \ldots, \alpha_{0}$, and $\gamma$. So $\mathbf{A}_{n}$ is a subalgebra of $\mathbf{A}_{n}^{\prime}$. Now $A_{n}$ has as elements

$$
\alpha_{0}, \alpha_{1}, \ldots, \alpha_{n-1}, \beta_{0}, \beta_{1}, \ldots, \beta_{n}
$$

but all the other elements of $A_{n}$ have at least one entry that is 0 .

Let $\theta$ be the equivalence relation on $A_{n}$ which lumps into a single equivalence class all the $n$-tuples that have at least one entry that is 0 and that isolates into singleton classes all other $n$-tuples. It is easy to check that $\theta$ is a congruence relation of $\mathbf{A}_{n}$. Let $\mathbf{B}_{n}=\mathbf{A}_{n} / \theta$. Slightly abusing notation, we take the elements of $B_{n}$ to be 0 (for the single large congruence class) as well as $\alpha_{0}, \ldots, \alpha_{n-1}, \beta_{0}, \ldots, \beta_{n}$ (for the singleton congruence classes). Notice that $\mathbf{B}_{n}$ has $2 n+2$ elements and that it is also an automatic algebra. The algebra $\mathbf{B}_{6}$ is displayed in Figure 2. 


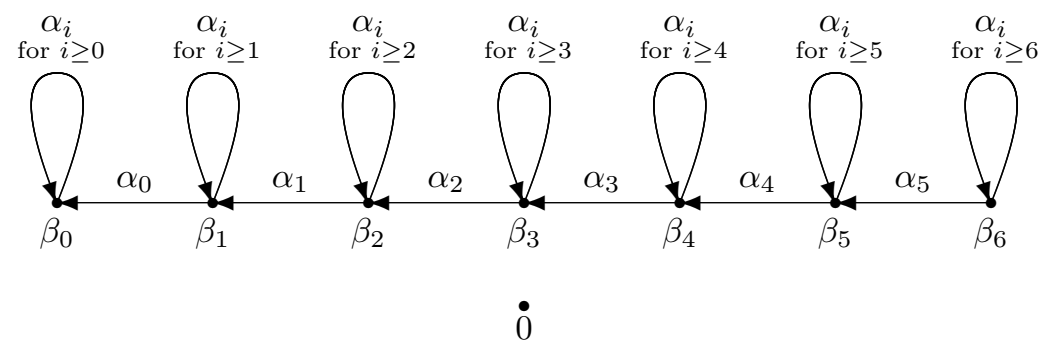

Figure 2. The Automatic Algebra $\mathbf{B}_{6}$

Most of the loops in Figure 2 have several labels; however, the loop drawn at $\beta_{6}$ is actually trivial in the sense that it has no label (it could be omitted, but was included for the sake of uniformity).

The algebra $\mathbf{A}_{n}^{\prime}$ has the congruence $\theta^{\prime}$ which collapses all the tuples that have at least one entry 0 into a single class and isolates the remaining elements of $A_{n}^{\prime}$. Let $\mathbf{B}_{n}^{\prime}=\mathbf{A}_{n}^{\prime} / \theta^{\prime}$. Observe that $\mathbf{B}_{n}$ is a subalgebra of $\mathbf{B}_{n}^{\prime}$ and that $\mathbf{B}_{n}^{\prime}$ has one more element than $\mathbf{B}_{n}$. Figure 3 displays $\mathbf{B}_{6}^{\prime}$. The diagram of $\mathbf{B}_{6}^{\prime}$

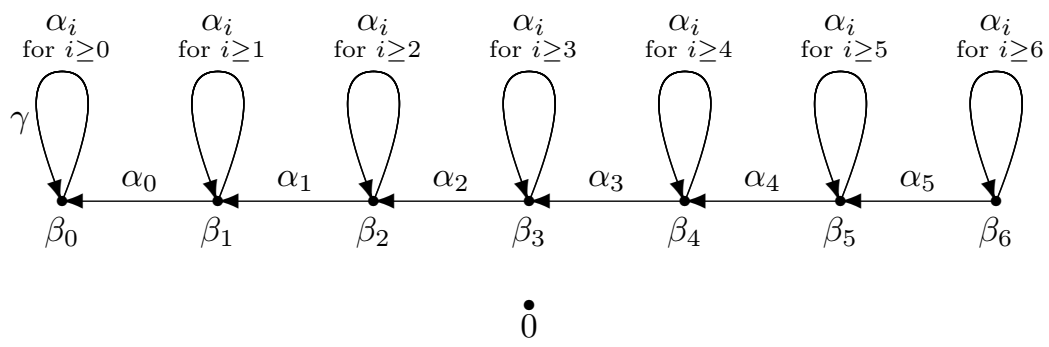

Figure 3. The Automatic Algebra $\mathbf{B}_{6}^{\prime}$

differs from that of $\mathbf{B}_{6}$ only by the attachment of the label $\gamma$ to the leftmost loop.

Since $\mathbf{L}^{\star}$ belongs to $\mathcal{V}$, we know that $\mathbf{B}_{n}, \mathbf{B}_{n}^{\prime} \in \mathcal{V}$ for all natural numbers $n$.

The algebra $\mathbf{C}_{n}$ is like $\mathbf{B}_{n}$ but with one more nontrivial product: $\beta_{n} \beta_{0}:=$ $\beta_{0}$. The algebra $\mathbf{C}_{n}$ is not an automatic algebra. We display $\mathbf{C}_{6}$ in Figure 4.

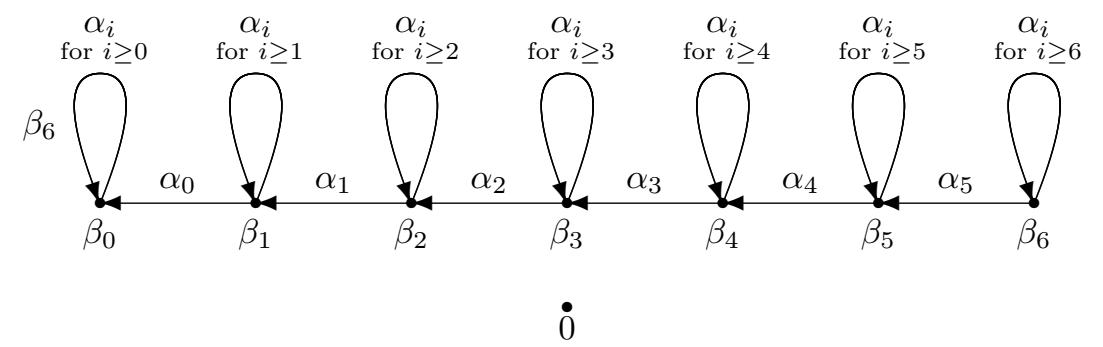

Figure 4. The Algebra $\mathbf{C}_{6}$ 
Claim 0. $\mathbf{C}_{n} \in \mathcal{V}^{(n)}$.

Proof. Consider any subalgebra $\mathbf{D}$ of $\mathbf{C}_{n}$ generated by any set of $n$ or fewer elements. Now none of $\beta_{n}, \alpha_{n-1}, \ldots, \alpha_{0}$ occur as outputs of the basic operation of $\mathbf{C}_{n}$. So unless these elements are in the generating set of $\mathbf{D}$ they cannot be in $\mathbf{D}$. In case $\beta_{n}$ is not in the generating set, we see that $\mathbf{D}$ is a subalgebra of $\mathbf{B}_{n}$. Hence $\mathbf{D} \in \mathcal{V} \subseteq \mathcal{V}^{(n)}$. In this case, $\mathbf{D} \in \mathcal{V}^{(n)}$ as desired. In case $\beta_{n}$ is in the generating set then one of the $\alpha_{i}$ 's must fail to be in the generating set and hence in $\mathbf{D}$. So suppose $\alpha_{j} \notin D$. Let $\mathbf{E}$ be the subalgebra of $\mathbf{C}_{n}$ obtained by omitting $\alpha_{j}$. This algebra is displayed in Figure 5.
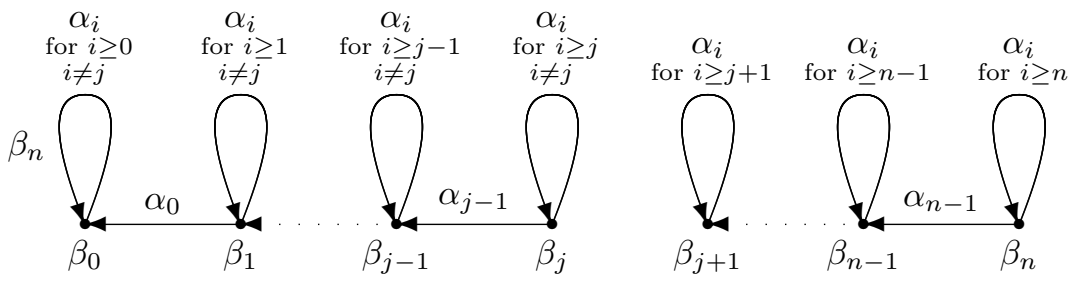

$\dot{0}$

Figure 5. The Algebra $\mathbf{E}$

We contend that $\mathbf{E}$ is a subalgebra of the direct product of two algebras, each belonging to $\mathcal{V}$. These algebras, which depend on $n$, are displayed in Figure 6.
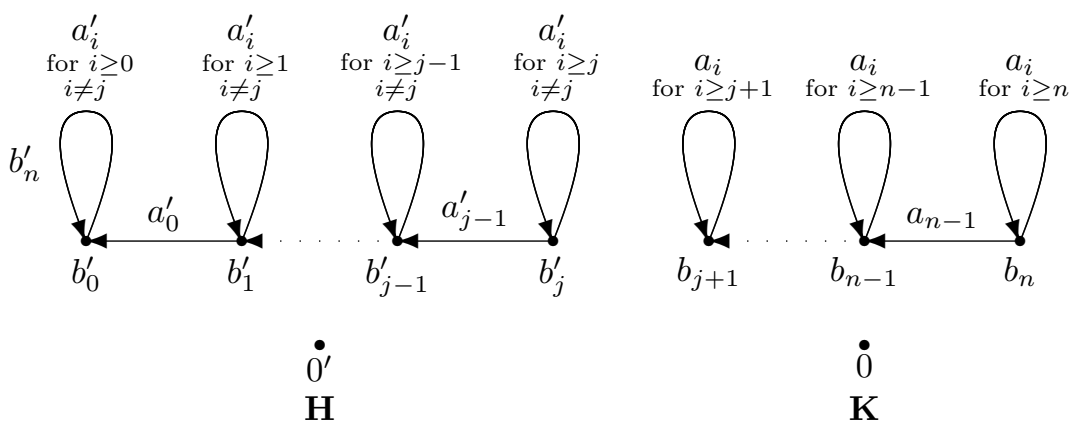

Figure 6. The Automatic Algebras $\mathbf{H}$ and $\mathbf{K}$

The elements of $\mathbf{K}$ are

$$
a_{j+1}, \ldots, a_{n-1}, b_{j+1} \ldots, b_{n} \text { and } 0,
$$

while the elements of $\mathbf{H}$ are

$$
a_{0}^{\prime}, \ldots, a_{j-1}^{\prime}, a_{j+1}^{\prime}, \ldots, a_{n-1}^{\prime}, b_{n}^{\prime}, b_{0}^{\prime}, \ldots, b_{j}^{\prime} \text { and } 0^{\prime} .
$$


The algebra $\mathbf{K}$ is seen to be isomorphic to a subalgebra of $\mathbf{B}_{n} \in \mathcal{V}$, while the algebra $\mathbf{H}$ is isomorphic to a subalgebra of $\mathbf{B}_{n}^{\prime} \in \mathcal{V}$. So $\mathbf{H}, \mathbf{K} \in \mathcal{V}$. The map that embeds $\mathbf{E}$ into $\mathbf{H} \times \mathbf{K}$ is given below:

$$
\begin{aligned}
\alpha_{i} & \mapsto\left(a_{i}^{\prime}, 0\right) \text { for } i<j \\
\alpha_{i} & \mapsto\left(a_{i}^{\prime}, a_{i}\right) \text { for } i>j \\
\beta_{i} & \mapsto\left(b_{i}^{\prime}, 0\right) \text { for } i \leq j \\
\beta_{i} & \mapsto\left(0, b_{i}\right) \text { for } n>i>j \\
\beta_{n} & \mapsto\left(b_{n}^{\prime}, b_{n}\right) \\
0 & \mapsto\left(0^{\prime}, 0\right)
\end{aligned}
$$

In this way we see that $\mathbf{E} \in \mathcal{V}$ and therefore every subalgebra of $\mathbf{C}_{n}$ with $n$ or fewer generators also belongs to $\mathcal{V}$. This means that $\mathbf{C}_{n} \in \mathcal{V}^{(n)}$, as the claim requires.

Claim 1. $\mathbf{C}_{n} \notin \mathcal{V}$.

Proof. For each natural number $n$, let $\epsilon_{n+1}$ be the following equation:

$$
y\left(x_{0}\left(x_{1} \ldots\left(x_{n-1} y\right) \ldots\right)\right) \approx y y .
$$

Each of these equations is easily seen to be true in every automatic algebra. In particular, each $\epsilon_{n+1}$ is true in $\mathbf{L}$ and hence in the variety $\mathcal{V}$. On the other hand, $\epsilon_{n+1}$ fails in $\mathbf{C}_{n}$ under the assignment that gives the value $\beta_{n}$ to the variable $y$ and, for each $i<n$, the value $\alpha_{i}$ to the variable $x_{i}$. Therefore $\mathbf{C}_{n} \notin \mathcal{V}$.

The two claims constitute this proof of Lyndon's Nonfinite Basis Theorem.

Lyndon's original proof also used the equations $\epsilon_{n+1}$ to separate $\mathcal{V}^{(n)}$ from $\mathcal{V}$.

The proof given above shows that Lyndon's algebra is actually inherently nonfinitely based relative to the class $\operatorname{Mod}\left\{\epsilon_{n+1} \mid n\right.$ is a natural number $\}$. This class includes all automatic algebras. So we obtain the following corollary.

Corollary. Lyndon's algebras is inherently nonfinitely based relative to the variety generated by the class of all automatic algebras.

In consequence, any automatic algebra that has $\mathbf{L}^{\star}$ as a subalgebra or a homomorphic image must also be nonfinitely based (and even inherently nonfinitely based relative to the variety generated by the class of all automatic algebras).

\section{Estimating the equational complexity of Lyndon's algebra}

Main Theorem. Let $\mathbf{L}$ be Lyndon's seven element nonfinitely based algebra. Then $n-4<\beta_{\mathbf{L}}(n) \leq 2 n+1$ for all $n>3$. 
Proof. To establish the first inequality we tailor, along the lines suggested in [20], the proof of Lemma 6 from [20] to our particular case.

Let $\mathcal{V}$ be the variety generated by $\mathbf{L}$ and let $k$ be a natural number. From the preceding section we know that $\mathbf{C}_{k} \notin \mathcal{V}$. So there is some equation true in $\mathbf{L}$ which fails in $\mathbf{C}_{k}$. Because $\mathbf{C}_{k} \in \mathcal{V}^{(k)}$, such an equation must involve at least $k+1$ distinct variables. Since our only operation symbol is binary, such an equation must have length at least $2 k$. (Evidently, such an equation must also have length at least 3 , so the lower bound of $2 k$ can be improved when $k=0$ or 1.) Since $\left|C_{k}\right|=2 k+2$ we obtain the inequality

$$
2 k<\beta_{\mathbf{L}}(2 k+2+1) \text { for all natural numbers } k .
$$

Now let $n$ be any natural number larger than 2. Fix the natural number $k$ so that

$$
2 k+3=(2 k+2)+1 \leq n<[2(k+1)+2]+1=2 k+5 .
$$

Combining the second inequality in $(\star)$ with $(*)$, we see that

$$
n-5<2 k<\beta_{\mathbf{L}}(2 k+3) \text {. }
$$

Since we are dealing with integer valued functions, we can conclude that

$$
n-4<\beta_{\mathbf{L}}(2 k+3) \text {. }
$$

On the other hand, from the monotonicity of $\beta_{\mathbf{L}}$ and the first inequality in $(\star)$, we get

$$
n-4<\beta_{\mathbf{L}}(2 k+3) \leq \beta_{\mathbf{L}}(n) \text { for all } n \geq 3 .
$$

In this way the first inequality of the theorem is established.

The next lemma was proven by Lyndon in [18].

Lyndon's Lemma. Let $\mathcal{V}$ be the variety generated by $\mathbf{L}$ and let $n \geq 3$ be a natural number. The following equations constitute an equational base for $\mathcal{V}^{(n)}$ :

$$
\begin{gathered}
(x y) z \approx x x \quad x(y y) \approx z z \\
y\left(x_{0}\left(x_{1} \ldots\left(x_{m-2} y\right) \ldots\right)\right) \approx y y \\
x_{m-2}\left(x_{0}\left(\ldots\left(x_{m-2} x_{m-1}\right) \ldots\right)\right) \approx x_{0}\left(\ldots\left(x_{m-2} x_{m-1}\right) \ldots\right)
\end{gathered}
$$

where $1<m \leq n$.

In his dissertation, John Boozer [4] observed that the equation $\delta_{3}$ has all the later $\delta_{n}$ has logical consequences. The following equations render $\delta_{2}$ and $\delta_{3}$ without subscripts:

$$
x(x y) \approx x y \quad x(z(x y)) \approx z(x y)
$$

Here is Boozer's reasoning illustrating a derivation of $\delta_{5}$ from $\delta_{3}$.

$$
\begin{aligned}
x_{0}\left(x_{1}\left(x_{2}\left(x_{3} x_{4}\right)\right)\right) & \approx x_{0}\left(x_{1}\left(x_{3}\left(x_{2}\left(x_{3} x_{4}\right)\right)\right)\right) \approx x_{0}\left(x_{3}\left(x_{1}\left(x_{3}\left(x_{2}\left(x_{3} x_{4}\right)\right)\right)\right)\right) \\
& \approx x_{3}\left(x_{0}\left(x_{3}\left(x_{1}\left(x_{3}\left(x_{2}\left(x_{3} x_{4}\right)\right)\right)\right)\right)\right) \approx x_{3}\left(x_{0}\left(x_{1}\left(x_{3}\left(x_{2}\left(x_{3} x_{4}\right)\right)\right)\right)\right) \\
& \approx x_{3}\left(x_{0}\left(x_{1}\left(x_{2}\left(x_{3} x_{4}\right)\right)\right)\right)
\end{aligned}
$$


This gives us

Boozer's Adjustment of Lyndon's Lemma. Let $\mathcal{V}$ be the variety generated by $\mathbf{L}$ and let $n \geq 3$ be a natural number. The following equations constitute an equational base for $\mathcal{V}^{(n)}$ :

$$
\begin{gathered}
(x y) z \approx x x \quad x(y y) \approx z z \quad x(x y) \approx x y \quad x(z(x y)) \approx z(x y) \\
y\left(x_{0}\left(x_{1} \ldots\left(x_{m-2} y\right) \ldots\right)\right) \approx y y \quad\left(\epsilon_{m}\right)
\end{gathered}
$$

where $1<m \leq n$.

We use Boozer's adjustment of Lyndon's Lemma to obtain an upper bound on $\beta_{\mathbf{L}}(n)$. Fix the number $n$. Consider all the algebras $\mathbf{B} \notin \mathcal{V}$ such that $|B|<n$. There must be a shortest equation true in $\mathbf{L}$ which fails in $\mathbf{B}$. We need an upper bound on the length of such equations. Consider a shortest equation belonging to the Boozer-Lyndon basis for $\mathbf{L}$ which fails in $\mathbf{B}$. An upper bound of $2 n$ on the length of such an equation will serve our purpose. We consider three cases:

\section{Case: It is one of the first three of the Boozer-Lyndon equations.}

The first three equations have length 8 , so 8 would serve as an upper bound in this case. Notice that if $3<n$, then $8 \leq 2 n$. So $2 n$ is also an upper bound. Case: It is the equation $x(z(x y)) \approx z(x y)$.

This equation has length 12 . So 12 would serve as an upper bound, but we want to determine how it compares with $2 n$.

In this case, we know that the first three equations of the Boozer-Lyndon basis hold in $\mathbf{B}$. This means that $\mathbf{B}$ must have an absorbing element which we denote by 0 . Now let $z \mapsto b_{0}, x \mapsto b_{1}$, and $y \mapsto b_{2}$ be an assignment of elements of $B$ to the variables in $x(z(x y)) \approx z(x y)$ which witnesses the failure of this equation. The right side of the equation is a subterm of the left side. It follows that the right side must evaluate to a non-0 element under our assignment. The evaluation trees for the equation under this asignment are displayed in Figure 7 .

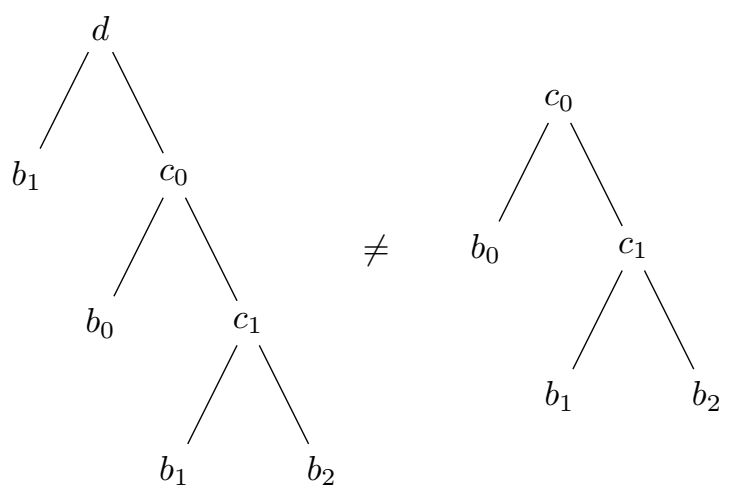

FigurE 7 . Evaluation trees of the equation $x(z(x y)) \approx z(x y)$ 
We claim the elements $b_{0}, b_{1}, b_{2}, c_{0}, c_{1}$ are all distinct (and all different from 0 , as is easily seen). We see that $b_{0} \neq b_{1}$ since otherwise our failure of the fourth Boozer-Lyndon equation would lead to a failure of the third. Now observe that $c_{0}=c_{1}$ would allow us to eliminate both $b_{0}$ and $c_{0}$ in Figure 7 to obtain a failure of the third of the Boozer-Lyndon equations. Finally, suppose to the contrary that $b_{i}=c_{j}$ for some $i, j<2$. Now we know that $c_{j}=b_{j} c_{j+1}$ (unless $j=1$ in which case $c_{1}=b_{1} b_{2}$ ). We also know that $b_{i} c_{i+1}=c_{i}$ (unless $i=1$ in which case $b_{1} b_{2}=c_{1}$ ). Since we assumed $b_{i}=c_{j}$, this gives us one the following possibilities:

$$
\begin{array}{ll}
\left(b_{0} c_{1}\right) c_{1}=c_{0} \neq 0 & \text { in case } b_{0}=c_{0} \\
\left(b_{1} b_{2}\right) c_{1}=c_{0} \neq 0 & \text { in case } b_{0}=c_{1} \\
\left(b_{0} c_{1}\right) b_{2}=c_{1} \neq 0 & \text { in case } b_{1}=c_{0} \\
\left(b_{1} b_{2}\right) b_{2}=c_{1} \neq 0 & \text { in case } b_{1}=c_{1}
\end{array}
$$

But each of these four possibilities witnesses a failure of the first of the BoozerLyndon equations, which we know holds in $\mathbf{B}$. Hence, the elements $b_{0}, b_{1}, c_{0}, c_{1}$ of $B$ are pairwise distinct (and all are different from 0 ).

Thus, $B$ has at least 5 elements. Hence $5<n$ and so $12 \leq 2 n$. Recall that 12 is the length of the fourth Boozer-Lyndon equation. In this case, we can use $2 n$ as an upper bound.

\section{Case: It is the equation $\epsilon_{k}$.}

We can assume that $\mathbf{B}$ is a model of the first two of the Boozer-Lyndon equations, so that $\mathbf{B}$ has an absorbing element 0 . Any element different from 0 generates 0 . This means that 0 is not needed in any generating set of $\mathbf{B}$ (unless $B$ has only one element). In particular, we know that $\mathbf{B}$ has a generating set of size no more than $n-2$. This means that $\mathbf{B} \notin \mathcal{V}^{(n-2)}$. This means that $k \leq n-2$. The length of $\epsilon_{n-2}$ is $2 n$. In this case, we can use $2 n$ as an upper bound as well. The referee pointed that the reasoning used in the previous case could have been used in this case, but the resulting bound would have been $4 n$ rather than $2 n$. We thank the referee.

Gathering these results together, we see that, as long as $n>3$, there must be an equation of length at most $2 \mathrm{n}$ which holds in $\mathbf{L}$ but fails in $\mathbf{B}$. Therefore $\beta_{\mathbf{L}}(n) \leq 2 n+1$ for all $n>3$.

Thus, both inequalities required by the theorem hold.

$$
\text { Let }
$$

$$
\Delta=\{(x y) z \approx x x, x(y y) \approx z z\} \cup\left\{y\left(x_{0}\left(x_{1} \ldots\left(x_{m-2} y\right) \ldots\right)\right) \approx y y \mid 1<m\right\} .
$$

The Polynomial Membership Problem Theorem. Let $\mathcal{W}$ be a variety based on $\Delta$ together with finitely many additional equations. The Finite Algebra Membership Problem for $\mathcal{W}$ can be solved in nondeterministic logarithmic space, and hence in polynomial time. 
Proof. We give a direct proof of the polynomial time claim first as it gives more precise time complexity bounds than that obtained by converting from nondeterministic logarithmic space.

Let $\Gamma$ be a finite set of equations so that $\mathcal{W}$ is based on $\Delta \cup \Gamma$. Let $\ell$ be a natural number larger than the number of variables occurring in any equation belonging to $\Gamma \cup\{(x y) z \approx x x, x(y y) \approx z z\}$. Let $\mathbf{B}$ be a groupoid of cardinality $n$. To determine whether an equation belonging to $\Gamma \cup\{(x y) z \approx x x, x(y y) \approx$ $z z\}$ is true in $\mathbf{B}$ it is only necessary to evaluate each side of the equation on at most $n^{\ell}$ tuples of elements from $B$. Since there are a fixed finite number of such equations, there is a polynomial $p(x)$ so that determining whether all the equations in $\Gamma \cup\{(x y) z \approx x x, x(y y) \approx z z\}$ are true in $\mathbf{B}$ can be done in $p\left(n^{\ell}\right)$ steps. But it remains to check the infinite list $\epsilon_{2}, \epsilon_{3}, \ldots$ of equations.

Now we assume that $\mathbf{B} \models \Gamma \cup\{(x y) z \approx x x, x(y y) \approx z z\}$. It follows that $\mathbf{B}$ has an absorbing element that we denote by 0 . Let $a$ be any element of $B$. We say $a$ is reachable from $a$ in 0 steps. We will say that $c \in B$ is reachable from $a$ in one step provided there is $b \in B$ so that $b a=c$. More generally, for each natural number $k, c$ is reachable from $a$ in $k+1$ steps provided $b d=c$ for some $b \in B$ and some $d \in B$ that is reachable from $a$ in $k$ steps. Finally, we say that $c$ is reachable from $a$ provided $c$ is reachable from $a$ in some finite number of steps. Let $L_{a}$ denote the set of all elements reachable from $a$.

It is clear that $\mathbf{B} \models\left\{y\left(x_{0}\left(x_{1} \ldots\left(x_{m-2} y\right) \ldots\right)\right) \approx y y \mid 1<m\right\}$ if and only if for each $a, b \in B$ if $b \in L_{a}$, then $a b=0$; this property is obviously verifiable in polynomial time provided that for each $a \in B$, the list $L_{a}$ can be produced in polynomial time. To do this, consider the operation table of $\mathbf{B}$ as an $n \times n$ array with the rows labelled by the left inputs and the columns labelled by the right inputs. We list $a$ first in $L_{a}$ since it is reachable in 0 steps. The elements reachable from $a$ in one step are exactly the entries in the column labelled by $a$. We list those elements next, omitting $a$ if it occurs. The elements reachable from $a$ in two steps are the entries in the columns labelled by the elements reachable from $a$ is one step. And so on. By organizing this process so that no column is examined more than once, we see that $L_{a}$ can be completely constructed by visiting each of the $n^{2}$ entries in the table no more than once. The task of ensuring that no column is visited more than once and that no element is listed more than once takes fewer than an additional $n^{2}$ decisions.

In itself, the method described above for determining whether $\mathbf{B} \in \mathcal{W}$, is not quite adequate. However, there is a well-known technique for completing such a line of reasoning. The trouble is that we have supposed that the elements of $B$ have complexity 1 . So reading in an element to the algorithm takes only one step of computation. To remedy this, we regard the elements of $B$ as strings of a fixed length over a two element alphabet. We take this fixed length to be $\lceil\log n\rceil$. In implementing our method, at various places it is convenient to have a linear order on $B$. We have the lexicographic order at our disposal. Determining if one element is less than another or if two elements are identical can be accomplished in about $\log n$ steps. The complexity of $\mathbf{B}$ is 
the complexity of its operation table, an $n \times n$ array whose entries are binary strings of length $\lceil\log n\rceil$.

Finally we sketch the proof of the (possibly) stronger claim that membership in $\mathcal{W}$ can be solved in nondeterministic logarithmic space $(\mathrm{NL})$. We use the fact that the Reachability problem in directed graphs is in NL (see [10, §3.3]) and that NL $=$ coNL (Immerman [9] and Szelepcsényi [29]). Consider a finite groupoid $\mathbf{B}$ that is not in $\mathcal{W}$; either $\Gamma$ fails on $\mathbf{B}$ or there is $b \in L_{a}$ with $a b \neq 0$. On a nondeterministic Turing machine with input algebra $\mathbf{B}$, first check validity of $\Gamma$ : as this is a first order property, it can be checked in logarithmic space. If $\Gamma$ fails then $\mathbf{B}$ is successfully found to be not in $\mathcal{W}$. Otherwise, nondeterministically write two elements $a, b \in B$ to the work tape and check that $b \in L_{a}$ with $a b \neq 0$; if so,then again $\mathbf{B}$ is discovered to be not in $\mathcal{W}$. The property $b \in L_{a}$ is an instance of the Reachability problem in the directed graph whose vertices are the elements of $B$ with edges corresponding to the "reachability in one step property" defined earlier in the proof. As Reachability is in NL and verifying $a b \neq 0$ can be achieved in logarithmic space, we find that verifying $\mathbf{B} \notin \mathcal{W}$ is in the complexity class NL.

In his dissertation [4], John Boozer proves that $\Delta$ is a base for the variety generated by the class of automatic algebras. So another way to state the theorem above is

Each variety that is finitely based relative to the variety generated by all automatic algebras has a finite algebra membership problem that is solvable in polynomial time (and even in nondeterministic logarithmic space).

Boozer's adjustment of Lyndon's Lemma shows that Lyndon's algebra is based on $\Delta$ together with two additional equations. So

Corollary. Lyndon's algebra generates a variety with a polynomial time finite algebra membership problem (one that can even be solved in nondeterministic logarithmic space).

\section{Cardinalities of subdirectly irreducible algebras in $\mathcal{V}$}

At Oberwolfach in 1976 Bjarni Jónsson speculated that if $\mathcal{V}$ is a finitely generated variety of finite signature with a finite residual bound, then $\mathcal{V}$ is finitely based. Also in 1976, Robert E. Park, in his dissertation [23] written at UCLA under the direction of Kirby Baker, framed the same statement as a conjecture. It is still open. Framed contrapositively we obtain

The Jónsson-Park Problem

Does every finitely generated nonfinitely based variety of finite signature have arbitrarily large finite subdirectly irreducible algebras? 
(The alternative of an infinite subdirectly irreducible algebra can be omitted, in view of a theorem of Robert W. Quackenbush [26] — cf. Wiesław Dziobiak [7].)

In essence, only five finite but nonfinitely based algebras (including $\mathbf{L}$ ) were known at the time Park was writing his dissertation, the fifth supplied by Park himself. (The year Park's dissertation was completed, the number of known and essentially different examples became infinite, with the examples devised by Polin [25].) In the last chapter of his dissertation, Park demonstrated that each of these five finite algebras generate varieties with arbitrarily large finite subdirectly irreducible algebras. For the variety $\mathcal{V}$ generated by Lyndon's algebra $\mathbf{L}$ and each cardinal $\kappa \geq 2$, Park's examples can be described as $\left(\mathbf{L}^{\star}\right)^{\kappa} / \theta$ where $\theta$ is the congruence that isolates each $\kappa$-tuple of letters and each $\kappa$-tuple of states but collapses all other $\kappa$-tuples into a single congruence class. Park's algebras have cardinality $1+2^{\kappa}+3^{\kappa}$ for every cardinal $\kappa \geq 2$. The algebras $\mathbf{B}_{n} \in \mathcal{V}$ described in Section 2 are easily seen to be subdirectly irreducible (with critical pair $\left(\beta_{0}, 0\right)$ ) when $n \geq 2$. These algebras have cardinality $2 n+2$ and so differ from those constructed by Park.

There is a third way to construct subdirectly irreducible algebras in $\mathcal{V}$. For every cardinal $\kappa \geq 2$, we provide the algebra $\mathbf{S}_{\kappa} \in \mathcal{V}$. This algebra has $2 \kappa+2$ elements and is subdirectly irreducible. Figure 8 displays $\mathbf{S}_{5}$. For these

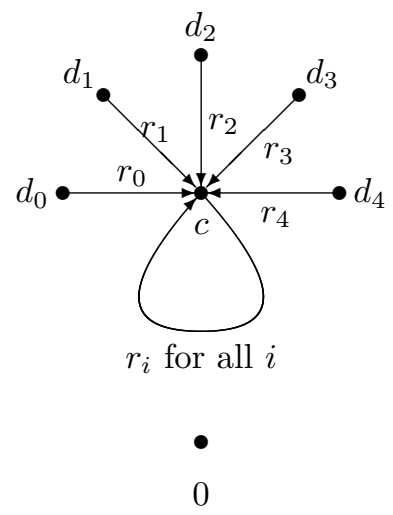

FiguRE 8. The Subdirectly Irreducible Automatic Algebra $\mathbf{S}_{5}$

algebras the pair $(c, 0)$ is critical. To see that these algebras are in $\mathcal{V}$, in $\left(\mathbf{L}^{\star}\right)^{\kappa}$ for each $\alpha \in \kappa$ put

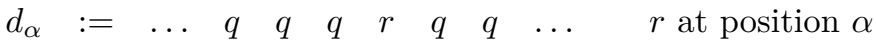

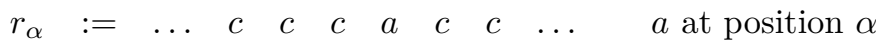

$$
\begin{aligned}
& c:=\ldots \begin{array}{lllllllllll}
c & := & \ldots & q & q & q & q & q & q & \ldots
\end{array}
\end{aligned}
$$


Let $\mathbf{T}_{\kappa}$ be the subalgebra generated by these elements. The equivalence relation $\theta_{\kappa}$ which places in one class all the tuples with at least one entry 0 and isolates all the other tuples is a congruence of $\mathbf{T}_{\kappa}$. We take $\mathbf{S}_{\kappa}$ to be $\mathbf{T}_{\kappa} / \theta_{\kappa}$.

To obtain finite subdirectly irreducible algebras of odd cardinality we modify $\mathbf{T}$ by including the tuple

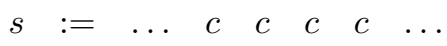

In this way, we obtain subdirectly irreducible algebras in $\mathcal{V}$ of every cardinality $\kappa \geq 6$. The algebra $\mathbf{L}^{\star}$ has subdirectly irreducible subalgebras of cardinalities 2,3 , and 5 which are displayed in Figure 9.

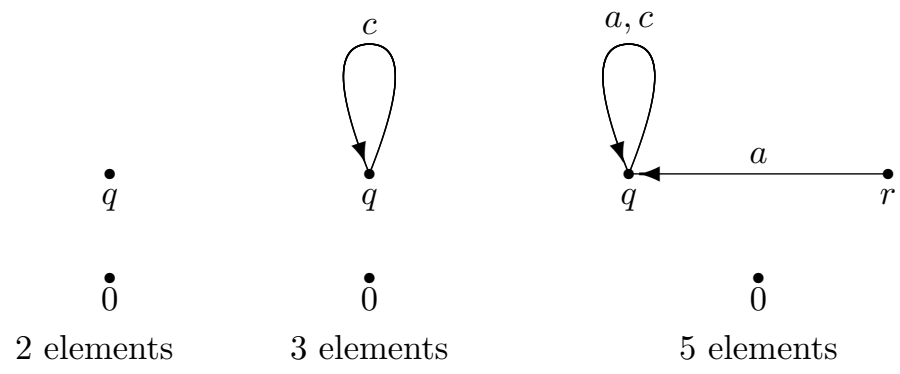

Figure 9. Three Small Subdirectly Irreducible Algebras

Up to isomorphism, $\mathcal{V}$ has just five algebras of cardinality 4 and none of them is subdirectly irreducible. These algebras, which are subalgebras of $\mathbf{L}$, are displayed in Figure 10.

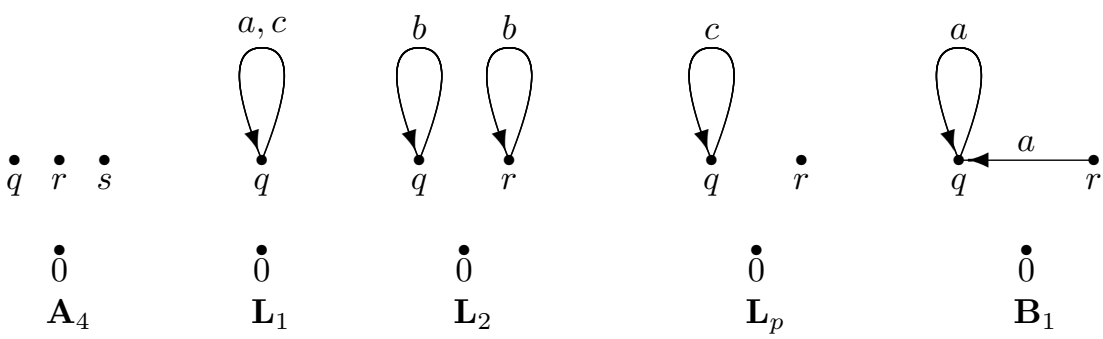

Figure 10. The 4-Element Algebras in Lyndon's Variety

To exclude the other possibilities, suppose the 4 elements are $0, a, b$, and $c$. For all $x, y, z \in\{0, a, b, c\}$, we have $0 x=x 0=0, x x=0,(x y) z=0, y(x y)=0$, and $x(x y)=x y$ because these equations are true in $\mathbf{L}$.

We can suppose that some product is not 0 , since if all products are 0 we get $\mathbf{A}_{4}$. It is harmless to suppose that $a b \neq 0$. It follows that $a$ cannot be a product, since if $a=u v$, the $0 \neq a b=(u v) b=0$. So there are two cases: $a b=c$ and $a b=b$.

First, suppose $a b=c$. Now $0=(a b) x=c x$ for all $x$. Also $a c=a(a b)=$ $a b=c$. Finally, $b c=b(a b)=0$. This leaves only the product $b a$ unsettled. If 
$b a=0$, we get the algebra $\mathbf{B}_{1}$. We already know it is impossible that $b a=a$. It is also impossible that $b a=b$ since this leads to $b=b a=(b a) a=0$. Finally, it is also impossible that $b a=c$ since this leads to $c=a c=a(b a)=0$.

Second, suppose that $a b=b$. Observe $0=(a b) x=b x$ for all $x$. We know $c a=a$ is impossible and $c a=b$ is impossible since $0=a(c a)=a b=b$ and $c a=c$ is impossible since $c=c a=(c a) a=0$. Therefore $c a=0$. In view of the first supposition, we can assume that $a c \neq b$. Because $a c=a$ is impossible, there are only two subcases: $a c=c$ and $a c=0$. If $a c=c$, then $0=(a c) x=c x$ for all $x$ and the resulting algebra is $\mathbf{L}_{2}$. So consider the remaining alternative $a c=0$. The only product left to consider is $c b$. It is impossible that $c b=c$ since then $c=c b=(c b) b=0$ and we know $c b=a$ is impossible. In case $c b=b$ we obtain the algebra $\mathbf{L}_{1}$ and in case $c b=0$ we obtain the algebra $\mathbf{L}_{p}$.

So we find that $\mathcal{V}$ has subdirectly irreducible algebras of every cardinality except 0,1 , and 4 . Ralph Freese has confirmed that $\mathcal{V}$ has no subdirectly irreducible algebras of cardinality 4 with the help of the Universal Algebra Calculator. This was accomplished by examining all 25 meet irreducible congruences of the algebra $\mathcal{V}$-freely generated by three elements. The Universal Algebra Calculator, originated by Matthew Valeriote, is largely the work of Ralph Freese and Emil Kiss. It is available online at http://www. uacalc.org/.

\section{The Shift Automorphism Theorem and Lyndon's algebra}

The theorem below can be used to show that a wide assortment of algebras are inherently nonfinitely based. It was published in 1989 by Kirby Baker, George McNulty, and Heinrich Werner [2]. An element $0 \in A$ is absorbing provided 0 is the output of any basic operation of $\mathbf{A}$ whenever 0 is among its inputs. All the elements of $A$ other than absorbing elements are said to be proper. A tuple of elements is proper if all of its entries are proper. A basic $r$-ary operation $F$ of $\mathbf{A}$ is a set of $r+1$-tuples.

The Shift Automorphism Theorem. Let A be an infinite locally finite algebra with only finitely many fundamental operations, with an absorbing element 0 , and with an automorphism $\sigma$ such that:

(a) $\{0\}$ is the only $\sigma$-orbit of $\mathbf{A}$ that is finite;

(b) the set of all proper tuples in $F$ is partitioned by $\sigma$ into only finitely many orbits, for each fundamental operation $F$ of $\mathbf{A}$;

(c) $f(a)=\sigma(a)$ for some proper element a of $A$ and some nonconstant unary polynomial function $f$ of $\mathbf{A}$.

Then $\mathbf{A}$ is inherently nonfinitely based.

Recently, two further conclusions have been added to the Shift Automorphism Theorem. Kate S. Owens [22] has shown that the variety generated by $\mathbf{A}$ has a countably infinite subdirectly irreducible algebra and McNulty, Székely and Willard [20] have proven that $\mathbf{A}$ is inherently nonfinitely based 
in the finite sense. This means that for any finitely based variety $\mathcal{U}$ to which A belongs there must be a natural number $p$ such that $\mathcal{U}$ has arbitrarily large finite $p$-generated algebras.

We know that the variety $\mathcal{V}$ generated by Lyndon's algebra fails to be inherently nonfinitely based. So no algebra in $\mathcal{V}$ can fulfill all the hypotheses of the Shift Automorphism Theorem. We construct an algebra $\mathbf{B}_{\mathbb{Z}} \in \mathcal{V}$ which fulfills all the conditions except for (b). This provides an example to show that the hypothesis (b) cannot be eliminated from the theorem (nor can it we replaced by the weaker condition that there be only finitely many $\sigma$-orbits).

The algebra $\mathbf{B}_{\mathbb{Z}}$ is displayed in Figure 11

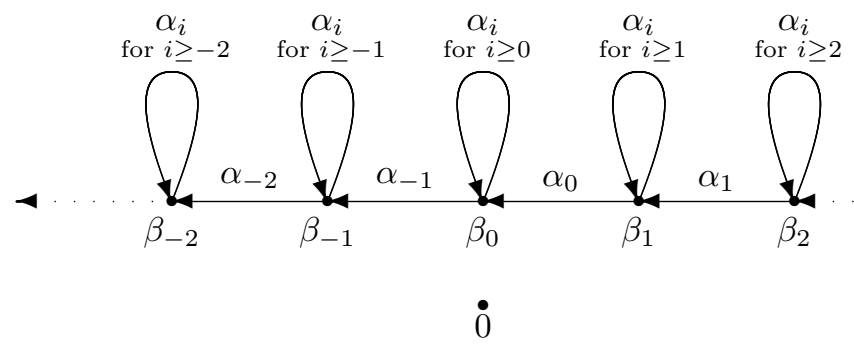

Figure 11. The Automatic Algebra $\mathbf{B}_{\mathbb{Z}}$

The algebra $\mathbf{B}_{\mathbb{Z}}$ is a homomorphic image of a subalgebra of $\left(\mathbf{L}^{\star}\right)^{\mathbb{Z}}$. The construction is much as the construction of $\mathbf{B}_{n}$ in Section 2. For each integer $k$ put

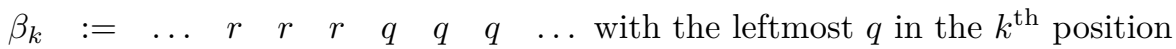

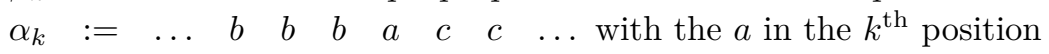

Let $\mathbf{G}$ be the subalgebra of $\left(\mathbf{L}^{\star}\right)^{\mathbb{Z}}$ generated by $\left\{\alpha_{k} \mid k \in \mathbb{Z}\right\} \cup\left\{\beta_{k} \mid k \in \mathbb{Z}\right\}$. The equivalence relation $\varphi$ on $G$ which puts all $\mathbb{Z}$-tuples with at least one entry 0 into one big equivalence class and isolates all the other tuples of $G$ is a congruence of $\mathbf{G}$. The algebra $\mathbf{B}_{\mathbb{Z}}$ is $\mathbf{G} / \varphi$. The map $\sigma$ that sends each $\beta_{k+1}$ to $\beta_{k}$, that sends each $\alpha_{k+1}$ to $\alpha_{k}$ and that fixes 0 is easily seen to be an automorphism of $\mathbf{B}_{\mathbb{Z}}$. This automorphism partitions $B_{\mathbb{Z}}$ into three orbits: the trivial orbit $\{0\}$, two infinite orbits $\left\{\alpha_{k} \mid k \in \mathbb{Z}\right\}$ and $\left\{\beta_{k} \mid k \in \mathbb{Z}\right\}$. Condition (a) of the Shift Automorphism Theorem is fulfilled. Observe that $\sigma\left(\beta_{1}\right)=\beta_{0}=\alpha_{0} \beta_{1}$. Hence the polynomial function $f(x)$ and the proper element $a$ required by hypothesis (c) of the Shift Automorphism Theorem can be taken as $\alpha_{0} x$ and $\beta_{1}$ respectively. However, hypothesis (b) fails: the triples $\left(\alpha_{k}, \beta_{0}, \beta_{0}\right)$ for $k \geq 0$ must all lie in distinct orbits.

\section{REFERENCES}

[1] Bajusz, T, McNulty, G. F., Szendrei, Á.: Lyndon's groupoid is not inherently nonfinitely based. Algebra Universalis 27, 254-260 (1990)

[2] Baker, K. A., McNulty, G. F., Werner, H.: Shift-automorphism methods for inherently nonfinitely based varieties of algebras. Czechoslovak Math. J. 39(114), 53-69 (1989) 
[3] Birkhoff, G.: On the structure of abstract algebras. Proc. Camb. Phil. Soc. 31, 433-454 (1935)

[4] Boozer, J.: On the Finite Axiomatizability of Equational Theories of Automatic Algebras. Ph.D. Thesis, University of South Carolina (2010)

[5] Cacioppo, R.: Non-finitely based pseudovarieties and inherently non-finitely based varieties. Semigroup Forum 47, 223-226 (1993)

[6] Clark, D. M., Davey, Brian A.: Natural dualities for the working algebraist. Cambridge Studies in Advanced Mathematics, vol. 57, Cambridge University Press, Cambridge, (1998)

[7] Dziobiak, W.: On infinite subdirectly irreducible algebras in locally finite equational classes. Algebra Universalis 13, 393-394 (1981)

[8] Eilenberg, S., Schützenberger, M. P.: On pseudovarieties. Advances in Math. 19, 413-418 (1976)

[9] Immerman, N.: Nondeterministic space is closed under complement. SIAM J. Comput. 17, 935-938 (1988)

[10] Immerman, N.: Descriptive Complexity. Advances in Mathematics, vol. 17. Springer-Verlag, New York (1999)

[11] Jackson, M.: Flat algebras and the translation of universal Horn logic to equational logic. J. Symbolic Logic 73, 90-128 (2008)

[12] Jackson, M., Volkov, M.: Relatively inherently nonfinitely q-based varieties of semigroups. Trans. Amer. Math. Soc. 361, 2181-2206 (2009)

[13] Kadourek, J.: On bases of identities of finite inverse semigroups with solvable subgroups. Semigroup Forum 67, 317-343 (2003)

[14] Kearnes, K.A., Willard, R.: Inherently nonfinitely based solvable algebras. Canad. Math. Bull. 37, 514-521 (1994)

[15] Kozik, M.: On Some Complexity Problems in Finite Algebras. Ph.D. Thesis, Vanderbilt University (2004)

[16] Kun, G., Vértesi, V.: The membership problem in finite flat hypergraph algebras. Internat. J. Algebra Comput. 17, 449-459 (2007)

[17] Lee, E. W. H.: Lyndon's groupoid generates a small almost Cross variety. Algebra Universalis 60, 239-246 (2009)

[18] Lyndon, R. C.: Identities in finite algebras. Proc. Amer. Math. Soc. 5, 8-9 (1954)

[19] McKenzie, R.: The residual bounds of finite algebras. Internat. J. Algebra Comput. 6, $1-28(1996)$

[20] McNulty, G. F., Székely, Z., Willard, R.: Equational complexity of the finite algebra membership problem. Internat. J. Algebra Comput. 18, 1283-1319 (2008)

[21] Murskiı̌, V.: Direct multiplication of finite algebras does not preserve finite equational bases: two examples and a general statement. Demonstratio Math. 29, 667-672 (1996)

[22] Owens, K. S.: Every shift automorphism variety has an infinite subdirectly irreducible member. J. Austral. Math. Soc. 88, 231-238 (2010)

[23] Park, R. E.: Equational Classes of Non-associative Ordered Algebras. Ph.D. Thesis, University of California, Los Angeles (1976)

[24] Pitkethly, J., Davey, B.: Dualisability, Unary Algebras and Beyond, Advances in Mathematics, vol. 9, Springer-Verlag, New York, (2005)

[25] Polin, S. V.: Identities of finite algebras. Sibirsk. Mat. Ž. 17, 1356-1366, 1439 (1976) (Russian)

[26] Quackenbush, R. W.: Equational classes generated by finite algebras. Algebra Universalis 1, 265-266 (1971/72)

[27] Sapir, M. V.: Sur la propriété de base finie pour les pseudovariétés de semigroupes finis. C. R. Acad. Sci. Paris Sér. I Math. 306, 795-797 (1988) (French, with English summary)

[28] Székely, Z.: Complexity of the Finite Algebra Membership Problem for Varieties. Ph.D. Thesis, University of South Carolina (1998)

[29] Szelepcsényi, R.: The method of forced enumeration for nondeterministic automata. Acta Inform. 26, 279-284 (1988) 
[30] Volkov, M. V.: The finite basis problem for finite semigroups. Sci. Math. Jpn. 53, 171-199 (2001)

MARCEL JACKSON

Department of Mathematics and Statistics, La Trobe University, Victoria, 3086, Australia.

e-mail: m.g.jackson@latrobe.edu.au

URL: http://marceljackson.ltumathstats.com/

George F. McNulty

Department of Mathematics, University of South Carolina, Columbia, SC 29208, USA. e-mail: mcnulty@math.sc.edu

$U R L:$ http://www.math.sc.edu/ mcnulty

DOI: $10.1007 / \mathrm{s} 00012-011-0126-3$

The final publication is available at http://www.springlink.com 